\title{
On the order of unimodular matrices modulo integers
}

\author{
by \\ PÄr KuRLBERG (Gothenburg)
}

1. Introduction. Given an integer $b$ and a prime $p$ such that $p \nmid b$, let $\operatorname{ord}_{p}(b)$ be the multiplicative order of $b$ modulo $p$. In other words, $\operatorname{ord}_{p}(b)$ is the smallest nonnegative integer $k$ such that $b^{k} \equiv 1 \bmod p$. Clearly $\operatorname{ord}_{p}(b) \leq$ $p-1$, and if the order is maximal, $b$ is said to be a primitive root modulo $p$. Artin conjectured (see the preface in [1]) that if $b \in \mathbb{Z}$ is not a square, then $b$ is a primitive root for a positive proportion $\left({ }^{1}\right)$ of the primes.

What about the "typical" behaviour of $\operatorname{ord}_{p}(b)$ ? For instance, are there good lower bounds on $\operatorname{ord}_{p}(b)$ that hold for a full density subset of the primes? In [3], Erdős and Murty proved that if $b \neq 0, \pm 1$, then there exists a $\delta>0$ so that $\operatorname{ord}_{p}(b)$ is at least $p^{1 / 2} \exp \left((\log p)^{\delta}\right)$ for a full density subset of the primes $\left({ }^{2}\right)$. However, we expect the typical order to be much larger. In [6] Hooley proved that the Generalized Riemann Hypothesis (GRH) implies Artin's conjecture. Moreover, if $f: \mathbb{R}^{+} \rightarrow \mathbb{R}^{+}$is an increasing function tending to infinity, Erdős and Murty [3] showed that GRH implies that the order of $b$ modulo $p$ is greater than $p / f(p)$ for a full density subset of the primes.

It is also interesting to consider lower bounds for $\operatorname{ord}_{N}(b)$ where $N$ is an integer. It is easy to see that $\operatorname{ord}_{N}(b)$ can be as small as $\log N$ infinitely often (take $N=b^{k}-1$ ), but we expect the typical order to be quite large. Assuming GRH, we can prove that the lower bound $\operatorname{ord}_{N}(b) \gg N^{1-\varepsilon}$ holds for most integers.

TheORem 1. Let $b \neq 0, \pm 1$ be an integer. Assuming GRH, the number of $N \leq x$ such that $\operatorname{ord}_{N}(b) \ll N^{1-\varepsilon}$ is o(x). That is, the set of integers $N$ such that $\operatorname{ord}_{N}(b) \gg N^{1-\varepsilon}$ has density one.

However, the main focus of this paper is to investigate a related question, namely lower bounds on the order of unimodular matrices modulo $N \in \mathbb{Z}$.

2000 Mathematics Subject Classification: Primary 11N37; Secondary 11A07.

Author supported in part by the National Science Foundation (DMS 0071503).

$\left({ }^{1}\right)$ The constant is given by an Euler product that depends on $b$.

$\left({ }^{2}\right)$ Pappalardi has shown [9] that $\delta$ can be taken to be approximately 0.15 . 
That is, if $A \in \mathrm{SL}_{2}(\mathbb{Z})$, what can be said about lower bounds for $\operatorname{ord}_{N}(A)$, the order of $A$ modulo $N$, that hold for most $N$ ? It is a natural generalization of the previous questions, but our main motivation comes from mathematical physics (quantum chaos): In [7] Rudnick and I proved that if $A$ is hyperbolic $\left({ }^{3}\right)$, then a strong form of quantum ergodicity for toral automorphisms follows from $\operatorname{ord}_{N}(A)$ being slightly larger than $N^{1 / 2}$, and we then showed that this condition holds for a full density subset of the integers $\left({ }^{4}\right)$. Again, we expect that the typical order is much larger. In order to give lower bounds on $\operatorname{ord}_{N}(A)$, it is essential to have good lower bounds on $\operatorname{ord}_{p}(A)$ for $p$ prime:

Theorem 2. Let $A \in \mathrm{SL}_{2}(\mathbb{Z})$ be hyperbolic, and let $f: \mathbb{R}^{+} \rightarrow \mathbb{R}^{+}$be an increasing function tending to infinity more slowly than $\log x$. Assuming $G R H$, there are at most $O\left(\frac{x}{f(x)^{1-\varepsilon} \log x}\right)$ primes $p \leq x$ such that $\operatorname{ord}_{p}(A)<$ $p / f(p)$. In particular, the set of primes $p$ such that $\operatorname{ord}_{p}(A) \geq p / f(p)$ has density one.

Using this we obtain an improved lower bound on $\operatorname{ord}_{N}(A)$ that is valid for most integers.

Theorem 3. Let $A \in \mathrm{SL}_{2}(\mathbb{Z})$ be hyperbolic. Assuming $G R H$, the number of $N \leq x$ such that $\operatorname{ord}_{N}(A) \ll N^{1-\varepsilon}$ is o(x). That is, the set of integers $N$ such that $\operatorname{ord}_{N}(A) \gg N^{1-\varepsilon}$ has density one.

Remarks. If $A$ is elliptic $(|\operatorname{tr}(A)|<2)$ then $A$ has finite order (in fact, at most 6). If $A$ is parabolic $(|\operatorname{tr}(A)|=2)$, then $\operatorname{ord}_{p}(A)=p$ unless $A$ is congruent to the identity matrix modulo $p$, and hence there exists a constant $c_{A}>0$ so that $\operatorname{ord}_{N}(A)>c_{A} N$. Apart from the application in mind, it is thus natural to only treat the hyperbolic case.

As far as unconditional results for primes go, we note that the proof in [3] relies entirely on analyzing the divisor structure of $p-1$, and we expect that their method should give a similar lower bound on the order of $A$ modulo $p$. An unconditional lower bound of the form

$$
\operatorname{ord}_{p}(b) \gg p^{\eta}
$$

for a full proportion of the primes and $\eta>1 / 2$ would be quite interesting. In this direction, Goldfeld [5] proved that if $\eta<3 / 5$, then (1) holds for a positive, but not full, proportion of the primes.

Clearly $\operatorname{ord}_{p}(A)$ is related to $\operatorname{ord}_{p}(\varepsilon)$, where $\varepsilon$ is one of the eigenvalues of $A$. Since $A$ is assumed to be hyperbolic, $\varepsilon$ is a power of a fundamental unit in a real quadratic field. The question of densities of primes $p$ such

$\left({ }^{3}\right) A$ is hyperbolic if $|\operatorname{tr}(A)|>2$.

$\left({ }^{4}\right)$ More precisely: there exists $\delta>0$ so that $\operatorname{ord}_{N}(A) \gg N^{1 / 2} \exp \left((\log N)^{\delta}\right)$ for a full density subset of the integers. 
that $\operatorname{ord}_{p}(\lambda)$ is maximal, for $\lambda$ a fundamental unit in a real quadratic field, does not seem to have received much attention until quite recently; in [10] Roskam proved that GRH implies that the set of primes $p$ for which ord $\operatorname{or}_{p}(\lambda)$ is maximal has positive density. (The work of Weinberger [12], Cooke and Weinberger [2] and Lenstra [8] does treat the case $\operatorname{ord}_{p}(\lambda)=p-1$, but not the case $\operatorname{ord}_{p}(\lambda)=p+1$.)

\section{Preliminaries}

2.1. Notation. If $\mathfrak{O}_{F}$ is the ring of integers in a number field $F$, we let $\zeta_{F}(s)=\sum_{\mathfrak{a} \subset \mathfrak{O}_{F}} N(\mathfrak{a})^{-s}$ denote the zeta function of $F$. By GRH we mean that all nontrivial zeros of $\zeta_{F}(s)$ lie on the line $\operatorname{Re}(s)=1 / 2$ for all number fields $F$.

Let $\varepsilon$ be an eigenvalue of $A$, satisfying the equation

$$
\varepsilon^{2}-\operatorname{tr}(A) \varepsilon+\operatorname{det}(A)=0 .
$$

Since $A$ is hyperbolic, $K=\mathbb{Q}(\varepsilon)$ is a real quadratic field. Let $\mathfrak{O}_{K}$ be the integers in $K$, and let $D_{K}$ be the discriminant of $K$. Since $A$ has determinant one, $\varepsilon$ is a unit in $\mathfrak{O}_{K}$. For $n \in \mathbb{Z}^{+}$we let $\zeta_{n}=e^{2 \pi i / n}$ be a primitive $n$th root of unity, and $\alpha_{n}=\varepsilon^{1 / n}$ be an $n$th root of $\varepsilon$. Further, with $Z_{n}=K\left(\zeta_{n}\right)$, $K_{n}=K\left(\zeta_{n}, \alpha_{n}\right)$, and $L_{n}=K\left(\alpha_{n}\right)$, we let $\sigma_{p}$ denote the Frobenius element in $\operatorname{Gal}\left(K_{n} / \mathbb{Q}\right)$ associated with $p$. We let $F_{p^{k}}$ denote the finite field with $p^{k}$ elements, and we let $F_{p^{2}}^{1} \subset F_{p^{2}}^{\times}$be the norm one elements in $F_{p^{2}}$, i.e., the kernel of the norm map from $F_{p^{2}}^{\times}$to $F_{p}^{\times}$. Let $\langle A\rangle_{p}$ be the group generated by $A$ in $\mathrm{SL}_{2}\left(F_{p}\right)$. Then $\langle A\rangle_{p}$ is contained in a maximal torus (of order $p-1$ or $p+1)$, and we let $i_{p}$ be the index of $\langle A\rangle_{p}$ in this torus. Finally, let $\pi(x)=\mid\{p \leq x: p$ is prime $\} \mid$ be the number of primes up to $x$.

2.2. Kummer extensions and Frobenius elements. We want to characterize primes $p$ such that $n \mid i_{p}$, and we can relate this to primes splitting in certain Galois extensions as follows:

Reduce equation (2) modulo $p$ and let $\bar{\varepsilon}$ denote a solution to equation (2) in $F_{p}$ or $F_{p^{2}}$. (Note that if $p$ does not ramify in $K$ then the order of $A$ modulo $p$ equals the order of $\varepsilon$ modulo $p$.) If $p$ splits in $K$ then $\bar{\varepsilon} \in F_{p}$, and if $p$ is inert, then $\bar{\varepsilon} \in F_{p^{2}} \backslash F_{p}$. In the latter case, $\bar{\varepsilon} \in F_{p^{2}}^{1}$ since the norm one property is preserved when reducing modulo $p$. Now, $F_{p}^{\times}$and $F_{p^{2}}^{1}$ are cyclic groups of order $p-1$ and $p+1$ respectively. Thus, if $p$ splits in $K$ then $\operatorname{ord}_{p}(\varepsilon) \mid p-1$, whereas if $p$ is inert in $K$ then $\operatorname{ord}_{p}(\varepsilon) \mid p+1$.

Lemma 4. Let $p$ be unramified in $K_{n}$, and let $C_{n}=\{1, \gamma\} \subset \operatorname{Gal}\left(K_{n} / \mathbb{Q}\right)$, where $\gamma$ is given by $\gamma\left(\zeta_{n}\right)=\zeta_{n}^{-1}$ and $\gamma\left(\alpha_{n}\right)=\alpha_{n}^{-1}$. Then the condition that $n \mid i_{p}$ is equivalent to $\sigma_{p} \in C_{n}$. Moreover, $C_{n}$ is invariant under conjugation. 
Proof. The split case: Since $n \mid i_{p}$ and $i_{p} \mid p-1$ we have $\zeta_{n} \in F_{p}$, i.e. $F_{p}$ contains all $n$th roots of unity. Moreover, $\bar{\varepsilon}$ is an $n$th power of some element in $F_{p}$, and thus the polynomial $x^{n}-\varepsilon$ splits completely in $F_{p}$. In other words, $p$ splits completely in $K_{n}$ and $\sigma_{p}$ is trivial.

The inert case: Since $n$ divides $i_{p}, \bar{\varepsilon}$ is an $n$th power of some element in $F_{p^{2}}^{1}$ and hence $\alpha_{n} \in F_{p^{2}}$. Moreover, $n \mid p^{2}-1$ implies that $\zeta_{n} \in F_{p^{2}}$. Now, $N_{F_{p}}^{F_{p^{2}}}\left(\alpha_{n}\right)=1$ and $N_{F_{p}}^{F_{p^{2}}}\left(\zeta_{n}\right)=\zeta_{n}^{p+1}=1$ implies that

$$
\sigma_{p}\left(\zeta_{n}\right) \equiv \zeta_{n}^{-1} \bmod p, \quad \sigma_{p}\left(\alpha_{n}\right) \equiv \alpha_{n}^{-1} \bmod p .
$$

For $p$ that does not ramify in $K_{n}$ we thus have

$$
\sigma_{p}\left(\zeta_{n}\right)=\zeta_{n}^{-1}, \quad \sigma_{p}\left(\alpha_{n}\right)=\alpha_{n}^{-1} .
$$

Now, an element $\tau \in \operatorname{Gal}\left(K_{n} / \mathbb{Q}\right)$ is of the form

$$
\tau: \begin{cases}\zeta_{n} \mapsto \zeta_{n}^{t}, & t \in \mathbb{Z}, \\ \alpha_{n} \mapsto \alpha_{n}^{u} \zeta_{n}^{s}, & s \in \mathbb{Z}, \quad u \in\{1,-1\} .\end{cases}
$$

Composing $\gamma$ and $\tau$ then gives

$$
\tau \circ \gamma:\left\{\begin{array}{l}
\zeta_{n} \mapsto \zeta_{n}^{-1} \mapsto \zeta_{n}^{-t}, \\
\alpha_{n} \mapsto \alpha_{n}^{-1} \mapsto \alpha_{n}^{-u} \zeta_{n}^{-s},
\end{array}\right.
$$

and

$$
\gamma \circ \tau:\left\{\begin{array}{l}
\zeta_{n} \mapsto \zeta_{n}^{t} \mapsto \zeta_{n}^{-t}, \\
\alpha_{n} \mapsto \alpha_{n}^{u} \zeta_{n}^{s} \mapsto \alpha_{n}^{-u} \zeta_{n}^{-s},
\end{array}\right.
$$

which shows that $\gamma$ is invariant under conjugation.

2.3. The Chebotarev Density Theorem. In [11] Serre proved that the Generalized Riemann Hypothesis (GRH) implies the following version of the Chebotarev Density Theorem:

Theorem 5. Let $E / \mathbb{Q}$ be a finite Galois extension of degree $[E: \mathbb{Q}]$ and discriminant $D_{E}$. For $p$ a prime let $\sigma_{p} \in G=\operatorname{Gal}(E / \mathbb{Q})$ denote the Frobenius conjugacy class, and let $C \subset G$ be a union of conjugacy classes. If the nontrivial zeros of $\zeta_{E}(s)$ lie on the line $\operatorname{Re}(s)=1 / 2$, then for $x \geq 2$,

$$
\left|\left\{p \leq x: \sigma_{p} \in C\right\}\right|=\frac{|C|}{|G|} \pi(x)+O\left(\frac{|C|}{|G|} x^{1 / 2}\left(\log D_{E}+[E: \mathbb{Q}] \log x\right)\right) .
$$

Now, primes that ramify in $K_{n}$ divide $n D_{K}$ (see Lemma 10), so as far as densities are concerned, ramified primes can be ignored. The bounds on the size of $D_{K_{n}}$ (see Lemma 10) and Lemma 4 then give the following:

Corollary 6. If GRH is true then

$$
\left|\left\{p \leq x: n \mid i_{p}\right\}\right|=\frac{2}{\left[K_{n}: \mathbb{Q}\right]} \pi(x)+O\left(x^{1 / 2}(\log (x n))\right) .
$$


Remark. For Theorems 2 and 3 to be true, it is enough to assume that the Riemann hypothesis holds for all $\zeta_{K_{n}}, n>1$.

2.3.1. Bounds on degrees. In order to apply the Chebotarev Density Theorem we need bounds on the degree $\left[K_{n}: \mathbb{Q}\right]$. We will first assume that $\varepsilon$ is a fundamental unit.

LEMma 7. If $\varepsilon$ is a fundamental unit in $K$ and if $n=4$ or $n=q$ for $q$ an odd prime, then $\operatorname{Gal}\left(K_{n} / K\right)$ is nonabelian.

Proof. We start by showing that $\left[K_{n}: Z_{n}\right]=n$. Consider first the case $n=q$. If $\alpha_{q} \in Z_{q}$ then $\beta=N_{K}^{Z_{q}}\left(\alpha_{q}\right)=\alpha_{q}^{\left[Z_{q}: K\right]} \zeta_{q}^{t} \in K \subset \mathbb{R}$ for some integer $t$. Since $q$ is odd we may assume that $\alpha_{q} \in \mathbb{R}$, and this forces $\zeta_{q}^{t}=1$, which in turn implies that $\alpha_{q}^{\left[Z_{q}: K\right]} \in K$. Because $\varepsilon$ is a fundamental unit this means that $q \mid\left[Z_{q}: K\right]$. On the other hand, $\left[Z_{q}: K\right] \mid \phi(q)$, a contradiction. Thus $\alpha_{q} \notin Z_{q}$, and hence $K_{q} / Z_{q}$ is a Kummer extension of degree $q$.

For $n=4$ we note that $i \in Z_{4}=K(i)$. Thus $\alpha_{2}=\sqrt{\varepsilon} \in Z_{4}$ implies that $\sqrt{-\varepsilon} \in Z_{4}$. However, either $\sqrt{\varepsilon}$ or $\sqrt{-\varepsilon}$ is real and generates a real degree two extension of $K$, whereas $K(i)$ is a nonreal quadratic extension of $K$, and hence $\alpha_{2} \notin Z_{4}$. Now, if $\alpha_{4} \in Z_{4}\left(\alpha_{2}\right)$ then $N_{Z_{4}}^{Z_{4}\left(\alpha_{2}\right)}\left(\alpha_{4}\right)=\alpha_{4}^{2} i^{t} \in Z_{4}$ for some $t \in \mathbb{Z}$, and thus $\alpha_{4}^{2}=\alpha_{2} \in Z_{4}$, which contradicts $\alpha_{2} \notin Z_{4}$. Therefore,

$$
\left[Z_{4}\left(\alpha_{4}\right): Z_{4}\right]=\left[Z_{4}\left(\alpha_{4}\right): Z_{4}\left(\alpha_{2}\right)\right]\left[Z_{4}\left(\alpha_{2}\right): Z_{4}\right]=4 .
$$

Finally, we note that the commutator of any nontrivial element $\sigma_{1} \in$ $\operatorname{Gal}\left(K_{n} / Z_{n}\right)$ with any nontrivial element $\sigma_{2} \in \operatorname{Gal}\left(K_{n} / L_{n}\right)$ is nontrivial (we may regard $\operatorname{Gal}\left(K_{n} / Z_{n}\right)$ and $\operatorname{Gal}\left(K_{n} / L_{n}\right)$ as subgroups of $\operatorname{Gal}\left(K_{n} / K\right)$ ). Hence $\operatorname{Gal}\left(K_{n} / K\right)$ is nonabelian.

LEMMA 8. If $\varepsilon$ is a fundamental unit then

$$
\left[K_{n}: Z_{n}\right] \geq n / 2 .
$$

Proof. Clearly $Z_{n}\left(\alpha_{q^{k}}\right) \subset K_{n}$, and since field extensions of relative prime degrees are disjoint, it is enough to show that if $q^{k} \| n$ is a prime power then $q^{k} \mid\left[Z_{n}\left(\alpha_{q^{k}}\right): Z_{n}\right]$ if $q$ is odd, and $q^{k-1} \mid\left[Z_{n}\left(\alpha_{q^{k}}\right): Z_{n}\right]$ if $q=2$.

If $q$ is odd then Lemma 7 implies that $\alpha_{q} \notin Z_{n}$ since $\operatorname{Gal}\left(Z_{n} / K\right)$ is abelian. Hence, if $m \in \mathbb{Z}$ and $\alpha_{q^{k}}^{m} \in Z_{n}$, we must have $q^{k} \mid m$. Now, if $\sigma \in \operatorname{Gal}\left(Z_{n}\left(\alpha_{q^{k}}\right) / Z_{n}\right)$ then $\sigma\left(\alpha_{q^{k}}\right)=\alpha_{q^{k}} \zeta_{q^{k}}^{t_{\sigma}}$ for some integer $t_{\sigma}$. Thus there exists an integer $t$ such that

$$
\beta=N_{Z_{n}}^{Z_{n}\left(\alpha_{q^{k}}\right)}\left(\alpha_{q^{k}}\right)=\alpha_{q^{k}}^{\left[Z_{n}\left(\alpha_{q^{k}}\right): Z_{n}\right]} \zeta_{q}^{t} \in Z_{n} .
$$

Multiplying $\beta$ by $\zeta_{q}^{-t} \in Z_{n}$ we find that $\alpha_{q^{k}}^{\left[Z_{n}\left(\alpha_{q^{k}}\right): Z_{n}\right]} \in Z_{n}$, and hence $q^{k} \mid\left[Z_{n}\left(\alpha_{q^{k}}\right): Z_{n}\right]$. 
For $q=2$ the proof is similar, except that a factor of two is lost if $\alpha_{2} \in Z_{n}$.

Remark. $K_{2} / \mathbb{Q}$ is a Galois extension of degree four, hence abelian and therefore contained in some cyclotomic extension by the Kronecker-Weber Theorem, and it is thus possible that $\alpha_{2} \in Z_{n}$ for some values of $n$.

Lemma 9. We have

$$
n \phi(n) \ll_{K}\left[K_{n}: \mathbb{Q}\right] \leq 2 n \phi(n) .
$$

Proof. We first observe that $\left[Z_{n}: K\right]$ equals $\phi(n)$ or $\phi(n) / 2$ depending on whether $K \subset \mathbb{Q}\left(\zeta_{n}\right)$ or not. We also have the trivial upper bound $\left[K_{n}: Z_{n}\right] \leq n$.

For a lower bound of $\left[K_{n}: Z_{n}\right]$ we argue as follows: Let $\gamma \in K$ be a fundamental unit. Since the norm of $\varepsilon$ is one we may write $\varepsilon=\gamma^{k}$ for some $k \in \mathbb{Z}$. (Note that $k$ does not depend on $n$.) As $\left[Z_{n}\left(\gamma^{1 / n}\right): Z_{n}\left(\varepsilon^{1 / n}\right)\right] \leq k$, Lemma 8 gives $\left[Z_{n}\left(\varepsilon^{1 / n}\right): Z_{n}\right] \geq n / k$. The upper and lower bounds now follow from

$$
\left[K_{n}: \mathbb{Q}\right]=\left[K_{n}: Z_{n}\right]\left[Z_{n}: K\right][K: \mathbb{Q}] .
$$

\subsubsection{Bounds on discriminants}

Lemma 10. If $p$ ramifies in $K_{n}$ then $p \mid n D_{K}$. Moreover,

$$
\log \left(\operatorname{disc}\left(K_{n} / \mathbb{Q}\right)\right) \ll_{K}\left[K_{n}: K\right] \log n .
$$

Proof. First note that

$$
\operatorname{disc}\left(K_{n} / \mathbb{Q}\right)=N_{\mathbb{Q}}^{K}\left(\operatorname{disc}\left(K_{n} / K\right)\right) \cdot \operatorname{disc}(K / \mathbb{Q})^{\left[K_{n}: K\right]} .
$$

From the multiplicativity of the different we get

$$
\operatorname{disc}\left(K_{n} / K\right)=\operatorname{disc}\left(Z_{n} / K\right)^{\left[K_{n}: Z_{n}\right]} \cdot N_{K}^{Z_{n}}\left(\operatorname{disc}\left(K_{n} / Z_{n}\right)\right) .
$$

Since $\varepsilon$ is a unit, so is $\varepsilon^{1 / n}$. Thus, if we let $f(x)=x^{n}-\varepsilon$ then $f^{\prime}(x)=n x^{n-1}$, and therefore the principal ideal $f^{\prime}\left(\varepsilon^{1 / n}\right) \mathfrak{O}_{K_{n}}$ equals $n \mathfrak{O}_{K_{n}}$. In terms of discriminants this means that

$$
\operatorname{disc}\left(K_{n} / Z_{n}\right) \mid N_{Z_{n}}^{K_{n}}\left(n \mathfrak{O}_{K_{n}}\right)
$$

and similarly it can be shown that

$$
\operatorname{disc}\left(Z_{n} / K\right) \mid N_{K}^{Z_{n}}\left(n \mathfrak{O}_{Z_{n}}\right) .
$$

Thus $\operatorname{disc}\left(K_{n} / \mathbb{Q}\right)$ divides

$$
\begin{aligned}
N_{\mathbb{Q}}^{K}\left(N_{K}^{K_{n}}\left(n \mathfrak{O}_{K_{n}}\right) \cdot N_{K}^{Z_{n}}\left(n \mathfrak{O}_{Z_{n}}\right)^{\left[K_{n}: Z_{n}\right]}\right) \cdot \operatorname{disc}(K / \mathbb{Q})^{\left[K_{n}: K\right]} & =n^{4\left[K_{n}: K\right]} \cdot \operatorname{disc}(K / \mathbb{Q})^{\left[K_{n}: K\right]},
\end{aligned}
$$

which proves the two assertions. 
3. Proof of Theorem 2. In order to bound the number of primes $p<x$ for which $i_{p}>x^{1 / 2}$ we will need the following lemma:

Lemma 11. The number of primes $p$ such that $\operatorname{ord}_{p}(A) \leq y$ is $O\left(y^{2}\right)$.

Proof. Given $A$ there exists a constant $C_{A}$ such that $\operatorname{det}\left(A^{n}-I\right)=$ $O\left(C_{A}^{n}\right)$. Now, if the order of $A$ modulo $p$ is $n$, then certainly $p$ divides $\operatorname{det}\left(A^{n}-I\right) \neq 0$. Putting $M=\prod_{n=1}^{y} \operatorname{det}\left(A^{n}-I\right)$ we see that any prime $p$ for which $A$ has order $n \leq y$ must divide $M$. Finally, the number of prime divisors of $M$ is bounded by

$$
\log M \ll \sum_{n=1}^{y} n \log C_{A} \ll y^{2} .
$$

First step: We consider primes $p$ such that $i_{p} \in\left(x^{1 / 2} \log x, x\right)$. By Lemma 11 the number of such primes is

$$
O\left(\left(\frac{x}{x^{1 / 2} \log x}\right)^{2}\right)=O\left(\frac{x}{\log ^{2} x}\right) .
$$

Second step: Consider $p$ such that $q \mid i_{p}$ for some prime $q \in\left(\frac{x^{1 / 2}}{\log ^{3} x}\right.$, $\left.x^{1 / 2} \log x\right)$. We may bound this by considering primes $p \leq x$ such that $p \equiv \pm 1 \bmod q$ for $q \in\left(\frac{x^{1 / 2}}{\log ^{3} x}, x^{1 / 2} \log x\right)$. Since $q \leq x^{1 / 2} \log x$, Brun's sieve gives (up to an absolute constant) the bound $x /(\phi(q) \log x)$, and the total contribution from these primes is at most

$$
\sum_{q \in\left(\frac{x^{1 / 2}}{\log ^{3} x}, x^{1 / 2} \log x\right)} \frac{x}{\phi(q) \log (x / q)} \ll \frac{x}{\log x} \sum_{q \in\left(\frac{x^{1 / 2}}{\log ^{3} x}, x^{1 / 2} \log x\right)} \frac{1}{q} .
$$

Now, summing reciprocals of primes in a dyadic interval, we get

$$
\sum_{q \in[M, 2 M]} \frac{1}{q} \ll \frac{\pi(2 M)}{M} \leq \frac{1}{\log M} .
$$

Hence

$$
\sum_{q \in\left(\frac{x^{1 / 2}}{\log ^{3} x}, x^{1 / 2} \log x\right)} \frac{1}{q} \ll \frac{1}{\log x} \log _{2}\left(\frac{x^{1 / 2} \log x}{x^{1 / 2} / \log ^{3} x}\right) \ll \frac{\log \log x}{\log x}
$$

and the right hand side of (6) is $O\left(\frac{x \log \log x}{\log ^{2} x}\right)$.

Third step: Now consider $p$ such that $q \mid i_{p}$ for some prime $q \in\left(f(x)^{2}\right.$, $\left.\frac{x^{1 / 2}}{\log ^{3} x}\right)$. We are now in the range where GRH is applicable; by Corollary 6 and Lemma 9 we have

$$
\left|\left\{p \leq x: q \mid i_{p}\right\}\right| \ll \frac{x}{q \phi(q) \log x}+O\left(x^{1 / 2} \log \left(x q^{2}\right)\right) .
$$


Summing over $q \in\left(f(x)^{2}, \frac{x^{1 / 2}}{\log ^{3} x}\right)$ we find that the number of such $p \leq x$ is bounded by

$$
\sum_{q \in\left(f(x)^{2}, \frac{x^{1 / 2}}{\log ^{3} x}\right)}\left(\frac{x}{q^{2} \log x}+O\left(x^{1 / 2} \log \left(x q^{2}\right)\right)\right) .
$$

Now,

$$
\sum_{q \in\left(f(x)^{2}, \frac{x^{1 / 2}}{\log ^{3} x}\right)} \frac{1}{q^{2}} \ll \frac{1}{f(x)}
$$

and thus (7) is

$$
\ll \frac{x}{f(x) \log x}+\frac{x}{\log ^{2} x} .
$$

Fourth step: For the remaining primes $p$, any prime divisor $q \mid i_{p}$ is smaller than $f(x)^{2}$. Hence $i_{p}$ must be divisible by some integer $d \in\left(f(x), f(x)^{3}\right)$. Again Lemmas 6 and 9 give

$$
\left|\left\{p \leq x: d \mid i_{p}\right\}\right| \ll \frac{x}{d \phi(d) \log x}+O\left(x^{1 / 2} \log \left(x d^{2}\right)\right) .
$$

Noting that $\phi(d) \gg d^{1-\varepsilon}$ and summing over $d \in\left(f(x), f(x)^{3}\right)$ we find that the number of such $p \leq x$ is bounded by

$$
\sum_{d \in\left(f(x), f(x)^{3}\right)}\left(\frac{x}{d^{2-\varepsilon} \log x}+O\left(x^{1 / 2} \log \left(x d^{2}\right)\right)\right) .
$$

Now,

$$
\sum_{d \in\left(f(x), f(x)^{3}\right)} \frac{1}{d^{2-\varepsilon}} \ll \frac{1}{f(x)^{1-\varepsilon}}
$$

and

$$
\sum_{d \in\left(f(x), f(x)^{3}\right)} x^{1 / 2} \log \left(x d^{2}\right) \ll f(x)^{3} x^{1 / 2} \log \left(x^{2}\right),
$$

therefore (8) is

$$
\ll \frac{x}{f(x)^{1-\varepsilon} \log x} .
$$

4. Proof of Theorems 1 and 3. Given a composite integer $N=$ $\prod_{p \mid N} p^{a_{p}}$ we wish to use the lower bounds on $\operatorname{ord}_{p}(b)\left(\operatorname{or}_{\operatorname{ord}}(A)\right)$ to obtain a lower bound on $\operatorname{ord}_{N}(b)$. The main obstacle is that $\operatorname{ord}_{N}(b)$ can be much smaller than $\prod_{p \mid N} \operatorname{ord}_{p^{a_{p}}}(b)$. Let $\lambda(N)$ be the Carmichael lambda function, i.e., the exponent of the multiplicative group $(\mathbb{Z} / N \mathbb{Z})^{\times}$. Clearly $\operatorname{ord}_{N}(b) \leq$ $\lambda(N)$, and it turns out that $\lambda(N)$ can be much smaller than $N$. However, 
$\lambda(N) \gg N^{1-\varepsilon}$ for most $N$ (see [4]), and since

$$
\operatorname{ord}_{N}(b) \geq \frac{\lambda(N)}{N} \prod_{p \mid N} \operatorname{ord}_{p}(b)
$$

it suffices to show that most integers are essentially given by a product of primes $p$ such that $\operatorname{ord}_{p}(b) \geq p / \log p$. We will only give the details for Theorem 3 since the other case is very similar.

If $p$ is prime such that $\operatorname{ord}_{p}(A) \leq p / \log p$, or $p$ ramifies in $K$, we say that $p$ is "bad". We let $P_{B}$ denote the set of all bad primes, and we let $P_{B}(z)$ be the set of primes $p \in P_{B}$ such that $p \geq z$. Since only finitely many primes ramify in $K$, Theorem 2 implies that the number of bad primes $p \leq x$ is $O\left(x / \log ^{2-\varepsilon} x\right)$. A key observation is the following:

LEMMA 12. We have

$$
\sum_{p \in P_{B}} \frac{1}{p}<\infty
$$

In particular, if we let

$$
\beta(z)=\sum_{p \in P_{B}(z)} 1 / p,
$$

then $\beta(z)$ tends to zero as $z$ tends to infinity.

Proof. Immediate from partial summation and the $O\left(x / \log ^{2-\varepsilon} x\right)$ estimate in Theorem 2.

Given $N \in \mathbb{Z}$, write $N=s^{2} N_{G} N_{B}$ where $N_{G} N_{B}$ is square-free and $N_{B}$ is the product of "bad" primes dividing $N$. By the following lemma, we find that few integers have a large square factor:

LEMMA 13. We have

$$
\left|\left\{N \leq x: s^{2} \mid N, s \geq y\right\}\right|=O(x / y) .
$$

Proof. The number of $N \leq x$ such that $s^{2} \mid N$ for $s \geq y$ is bounded by $\sum_{s \geq y} x / s^{2} \ll x / y$

Next we show that there are few $N$ for which $N_{B}$ is divisible by $p \in$ $P_{B}(z)$. In other words, for most $N, N_{B}$ is a product of small "bad" primes.

Lemma 14. The number of $N \leq x$ such that $p \in P_{B}(z)$ divides $N_{B}$ is $O(x \beta(z))$.

Proof. Let $p \in P_{B}(z)$. The number of $N \leq x$ such that $p \mid N$ is less than $x / p$. Thus, the total number of $N \leq x$ such that some $p \in P_{B}(z)$ divides $N$, is bounded by

$$
\sum_{p \in P_{B}(z)} \frac{x}{p}=x \sum_{p \in P_{B}(z)} \frac{1}{p}=x \beta(z) .
$$


Combining the previous results we find that the number of $N=s^{2} N_{G} N_{B}$ $\leq x$ such that $N_{B}$ is $z$-smooth and $s \leq y$ is

$$
x(1+O(\beta(z)+1 / y)) .
$$

For such $N$ we have $N_{B} \leq \prod_{p \leq z} p \ll e^{z}$. Letting $z=\log \log x$ and $y=\log x$ we get

$$
N_{G}=\frac{N}{s^{2} N_{B}} \geq \frac{N}{\log ^{3} x}
$$

for $N \leq x$ with at most $O\left(x\left(\beta(\log \log x)+(\log x)^{-1}\right)\right)=o(x)$ exceptions. Now, the following proposition shows that, for most $N$, $\operatorname{ord}_{N}(A)$ is essentially given by $\prod_{p \mid N} \operatorname{ord}_{p}(A)$.

Proposition ([7, Proposition 11]). Let $D_{A}=4\left(\operatorname{tr}(A)^{2}-4\right)$. For almost all $\left(^{5}\right) \quad N \leq x$

$$
\operatorname{ord}_{N}(A) \geq \frac{\prod_{p \mid d_{0}} \operatorname{ord}_{p}(A)}{\exp \left(3(\log \log x)^{4}\right)}
$$

where $d_{0}$ is given by writing $N=d s^{2}$, with $d=d_{0} \operatorname{gcd}\left(d, D_{A}\right)$ square-free.

Finally, $\operatorname{since} \operatorname{ord}_{p}(A) \geq p / \log p \geq p^{1-\varepsilon}$ for $p \mid N_{G}$ and $p$ sufficiently large, we find that

$$
\operatorname{ord}_{N}(A) \gg \frac{\prod_{p \mid N_{G}} \operatorname{ord}_{p}(A)}{\exp \left(3(\log \log x)^{4}\right)} \gg \frac{N_{G}^{1-\varepsilon}}{\exp \left(3(\log \log x)^{4}\right)} \gg N^{1-2 \varepsilon}
$$

for all but $o(x)$ integers $N \leq x$.

\section{References}

[1] E. Artin, The Collected Papers of Emil Artin, Addison-Wesley, Reading, 1965.

[2] G. Cooke and P. J. Weinberger, On the construction of division chains in algebraic number rings, with applications to $\mathrm{SL}_{2}$, Comm. Algebra 3 (1975), 481-524.

[3] P. Erdős and M. R. Murty, On the order of a $(\bmod p)$, in: Number Theory (Ottawa, ON, 1996), Amer. Math. Soc., Providence, RI, 1999, 87-97.

[4] P. Erdős, C. Pomerance and E. Schmutz, Carmichael's lambda function, Acta Arith. 58 (1991), 363-385.

[5] M. Goldfeld, On the number of primes $p$ for which $p+a$ has a large prime factor, Mathematika 16 (1969), 23-27.

[6] C. Hooley, On Artin's conjecture, J. Reine Angew. Math. 225 (1967), 209-220.

[7] P. Kurlberg and Z. Rudnick, On quantum ergodicity for linear maps of the torus, Comm. Math. Phys. 222 (2001), 201-227.

[8] H. W. Lenstra, Jr., On Artin's conjecture and Euclid's algorithm in global fields, Invent. Math. 42 (1977), 201-224.

[9] F. Pappalardi, On the order of finitely generated subgroups of $\mathbf{q}^{*}(\bmod p)$ and divisors of $p-1$, J. Number Theory 57 (1996), 207-222.

$\left({ }^{5}\right)$ By "for almost all $N \leq x$ " we mean that there are $o(x)$ exceptional integers $N$ that are smaller than $x$. 
[10] H. Roskam, A quadratic analogue of Artin's conjecture on primitive roots, ibid. 81 (2000), 93-109.

[11] J.-P. Serre, Quelques applications du théorème de densité de Chebotarev, Inst. Hautes Études Sci. Publ. Math. 54 (1981), 323-401.

[12] P. J. Weinberger, On Euclidean rings of algebraic integers, in: Analytic Number Theory, Proc. Sympos. Pure Math. 24 (St. Louis, MO, 1972), Amer. Math. Soc., Providence, RI, 1973, 321-332.

Department of Mathematics

Chalmers University of Technology

SE-412 96 Gothenburg, Sweden

E-mail: kurlberg@math.chalmers.se

URL: www.math.chalmers.se/ ${ }^{\sim}$ kurlberg 